\title{
High-resolution melting analysis reveals low Plasmodium parasitaemia infections among microscopically negative febrile patients in western Kenya
}

\author{
Purity N Kipanga ${ }^{1,3}$, David Omondi ${ }^{1,4}$, Paul O Mireji ${ }^{5}$, Patrick Sawa ${ }^{2}$, Daniel K Masiga ${ }^{1}$ and Jandouwe Villinger ${ }^{1 *}$
}

\begin{abstract}
Background: Microscopy and rapid diagnostic tests (RDTs) are common tools for diagnosing malaria, but are deficient in detecting low Plasmodium parasitaemia. A novel molecular diagnostic tool (nPCR-HRM) that combines the sensitivity and specificity of nested PCR (nPCR) and direct PCR-high resolution melting analysis (dPCR-HRM) was developed. To evaluate patterns of anti-malarial drug administration when no parasites are detected, nPCR-HRM was employed to screen blood samples for low parasitaemia from febrile patients without microscopically detectable Plasmodium infections in a rural malaria-endemic setting.
\end{abstract}

Methods: Blood samples $(n=197)$ were collected in two islands of Lake Victoria, Kenya, from febrile patients without Plasmodium detectable by microscopy or RDTs. 185 rRNA gene sequences were amplified from extracted DNA by $\mathrm{nPCR}-\mathrm{HRM}, \mathrm{nPCR}$, and $\mathrm{APCR}-\mathrm{HRM}$ to detect and differentiate Plasmodium parasites. The limits of detection (LoD) were compared using serial dilutions of the WHO International Standard for P. falciparum DNA. Data on administration of anti-malarials were collected to estimate prescription of anti-malarial drugs to patients with and without low parasitaemia Plasmodium infections.

Results: The coupled nPCR-HRM assay detected Plasmodium parasites with greater sensitivity (LoD $=236$ parasites $/ \mathrm{mL}$ ) than either $\mathrm{nPCR}$ ( $\mathrm{LoD}=4,700$ parasites $/ \mathrm{mL}$ ) or $\mathrm{dPCR}-\mathrm{HRM}$ ( $\mathrm{LoD}=1,490$ parasites $/ \mathrm{mL}$ ). Moreover, $\mathrm{nPCR}-\mathrm{HRM}$ detected and differentiated low-parasitaemia infections in significantly greater proportions of patients than did either nPCR or dPCR-HRM ( $p$-value $<0.001$ ). Among these low-parasitaemia infections, $67.7 \%$ of patients were treated with anti-malarials, whereas $81.5 \%$ of patients not infected with Plasmodium parasites were treated with anti-malarials.

Conclusions: The enhanced sensitivity of nPCR-HRM demonstrates limitations of differential febrile illness diagnostics in rural malaria endemic settings that confound epidemiological estimates of malaria, and lead to inadvertent misadministration of anti-malarial drugs. This is the first study that employs low-parasitaemia Plasmodium diagnostics to quantify the prescription of anti-malarial drugs to both non-malaria febrile patients and patients with low-parasitaemia Plasmodium infections. nPCR-HRM enhances low-parasitaemia malaria diagnosis and can potentially surmount the deficiencies of microscopy and RDT-based results in determining low-parasitaemia Plasmodium infection rates for evaluating malaria elimination efforts. The findings highlight the need for improved differential diagnostics of febrile illness in remote malaria endemic regions.

Keywords: Malaria misdiagnosis, antimalarial drug prescription, high resolution melting analysis (HRM), low-parasitaemia malaria detection, Plasmodium differentiation, febrile illness differential diagnostics

\footnotetext{
* Correspondence: jandouwe@icipe.org

'Martin Lüscher Emerging Infectious Diseases (ML-EID) Laboratory, Molecular Biology and Bioinformatics Unit, International Centre of Insect Physiology and Ecology (icipe), P.O. Box 30772, Nairobi 00100, Kenya

Full list of author information is available at the end of the article
} 


\section{Background}

The World Health Organization (WHO) estimates that despite recent reductions in malaria incidence, malaria contributed to the morbidity and mortality of about 166 million and 564,000 people, respectively, in sub-Saharan Africa (SSA) in 2012 [1]. However, other epidemiological estimates nearly double these figures [2]. It is unclear how 'presumptive diagnosis' has influenced these statistics [3], since patients presenting with febrile illnesses with undetected parasitaemia by microscopy or rapid diagnostic tests (RDTs) are inadvertently integrated into the statistics, confounding prevalence measures of malaria in the process [4-6].

Appropriate diagnosis is not only important for ensuring prudent use of anti-malarial medications, and facilitating correct prognosis based on appropriate differential diagnosis $[1,7,8]$, but also for tracking malaria elimination efforts [9-11]. Conventional microscopy [12] and RDT-based malaria diagnostics [13] used in malaria endemic regions of Africa have relatively low sensitivity and specificity in the detection of low-parasitaemia infections $[14,15]$. Although the limits of detection (LoD) of parasitaemia by microscopy (100 parasites/ $\mu \mathrm{L}$ or higher depending on technical skills) $[16,17]$ and RDT (50-400 parasites/ $\mu \mathrm{L})[18]$ may be well below the estimated parasite density threshold $(3,500$ parasites $/ \mu \mathrm{L}$ ) for initiation of febrile episodes [19], many clinicians in areas of high malaria endemicity are compelled to heuristically prescribe anti-malarials on the basis of clinical symptoms alone [6,7,20-22]. Such diagnostic and anti-malarial prescription practices in the absence of malaria positive microscopy and/or RDT results [6-8] may cause overlooking potentially severe non-malarial febrile illnesses that may coexist in malaria endemic tropical regions, such as bacterial, mycobacterial, fungal, and arthropod-borne viral infections [20,11,23,24]. Indeed, even reliance on RDTs, which may also detect sub-clinical Plasmodium parasitaemia, has been implicated in undermining considerations of such differential diagnoses [10].

While low-parasitaemia Plasmodium diagnostics may be inappropriate for attributing febrile symptoms to malaria, effective malaria elimination efforts must be able to identify sub-clinical, low-parasitaemia Plasmodium infections that contribute to the infectious reservoir [12]. Molecular techniques are generally superior to antigen-detection tests and microscopy for detecting pathogens in biological samples $[12,13,25,26]$. Specifically, techniques such as nested Polymerase chain reaction (nPCR) can enhance the sensitivity of detecting low-parasitaemia Plasmodium infections by increasing the total number of PCR cycles and by diluting the inhibitors present in the initial amplification reaction [27]. Similarly, direct PCR with high resolution melting (dPCR-HRM) analysis can distinguish between different Plasmodium species in a sample [28] and can hence provide better information on the relative abundance and composition of Plasmodium species in a study area.

This paper describes a novel molecular diagnostic technique that combines nested PCR with HRM analysis (nPCR-HRM) to detect and differentiate low-parasitaemia Plasmodium infections. The sensitivity of the novel coupled nPCR-HRM approach was compared to the performance of separate and independent implementations of nPCR [27] and APCR-HRM [28] assays. The novel technique was further applied to quantify misadministration of anti-malarial drugs among patients presenting febrile symptoms without Plasmodium infections detectable by microscopy or RDTs in rural clinics on Rusinga and Mfangano Islands of Lake Victoria in Kenya.

\section{Methods}

The study was approved by the National Ethical Review Board located at the Kenya Medical Research Institute, KEMRI (Approval Ref: Non-SSC Protocol \#310). Project staff explained the study to the participants in the local language and provided participants with study information. Written informed consent was obtained from all patients sampled with febrile illness. Adults accompanying patients between 12 and 17 years old consented on behalf of the patients.

\section{Study sites}

The study was conducted on the Lake Victoria islands of Mfangano (at Sena Health Centre, $0^{\circ} 28^{\prime} 24.10^{\prime \prime S} / 34^{\circ}$ 4'2.54"E) and Rusinga (at Tom Mboya Health Centre, $0^{\circ}$ $24^{\prime} 0.66^{\prime \prime S} / 34^{\circ} 9^{\prime} 50.52^{\prime \prime E}$ ) located in western Kenya. Both islands have a population of between 20,000 and 30,000 people, and are in the Mbita constituency of Homa Bay County, a malaria endemic region in western Kenya. This region has recorded malaria prevalence as high as 50\% between 2001 and 2004 [29]. Malaria transmission fluctuates with seasons, but is sustained throughout the year in the two islands. Anopheles gambiae, Anopheles arabiensis, and Anopheles funestus are the primary vectors of malaria [30] with Plasmodium falciparum as the most prevalent malaria parasite [31]. Rusinga Island is connected to the mainland via a 250-metre causeway constructed in the early 1980s through rock and earth filling. Mfangano Island is more isolated, located approximately $16 \mathrm{Km}$ from the mainland. Both Sena and Tom Mboya Health Centres rely heavily on microscopy for malaria diagnostics, and use RDT's inconsistently, as available. The area typically experiences long and short rainy seasons that are accompanied with an upsurge of malaria infections. The long rains extend from March to May and the short rains between August and December. Temperatures range from $17^{\circ} \mathrm{C}$ to $34^{\circ} \mathrm{C}$ with annual rainfall ranging between $700 \mathrm{~mm}$ to $1,200 \mathrm{~mm}$ [32]. 


\section{Human blood sampling}

Blood sampled from patients visiting the two health centres presenting with febrile symptoms (fever above $37.5^{\circ} \mathrm{C}$, headache, joint pains, diarrhoea, cough, abdominal pain, constipation, nausea) were screened for the presence of Plasmodium parasites by microscopy (thick blood smear) and RDT (CareStart ${ }^{\text {tm }}$ Malaria HRP2(Pf)). Patients who provided samples with detectable Plasmodium parasites using these approaches were treated for malaria, allowed to go home and were excluded from this study. Blood $(3 \mathrm{~mL})$ from consecutive RDT and smear-negative febrile patients was drawn from consenting patients (12 years and above). All patients that had taken anti-malarial drugs within the preceding two weeks were excluded from the study. Blood samples from patients were collected using heparinized vacutainer collection tubes (MacMed Healthcare), aliquoted into three separate cryovials, and immediately stored in liquid nitrogen shippers. Trained hospital personnel also filled out structured questionnaires pertaining to demographic and clinical data of patients. To minimize selection bias, only two patients per week from each clinic were included in the study. Filled shippers and questionnaires were transported back to the laboratory for analysis. To protect patient anonymity, all blood samples and questionnaires were labelled with barcode identifiers. All samples were collected between May 2012 and October 2013, facilitated by staff in the Division of Disease Surveillance and Response (DDSR) of the Kenyan Ministry of Public Health and Sanitation.

\section{Extraction of total DNA from blood}

Total DNA in blood samples were extracted by methods of Kawasaki [33] with minor modifications. Briefly, $50 \mu \mathrm{L}$ of blood samples were separately transferred from cryovials to respective $1.5 \mathrm{~mL}$ eppendorf tubes containing $0.5 \mathrm{~mL}$ of Tris-EDTA (TE) buffer $(10 \mathrm{mM}$ Tris- $\mathrm{HCl}$, $1 \mathrm{mM}$ EDTA; $\mathrm{pH}$ 8.0). The samples were vortexed for ten seconds and centrifuged for five minutes at 13,000 relative centrifugal force (rcf) at $4^{\circ} \mathrm{C}$. The resultant supernatants were discarded and the pellets were washed three times by re-suspending in the TE buffer, vortexing for ten seconds, and centrifuging for five minutes. The final pellets were re-suspended in $100 \mu \mathrm{L}$ of proteinase $\mathrm{K}$ buffer $\left(1.5 \mathrm{mM} \mathrm{MgCl}_{2}, 50 \mathrm{mM} \mathrm{KCl}, 0.5 \%\right.$ Tween 20 , $100 \mu \mathrm{g} / \mathrm{mL}$ proteinase $\mathrm{K}$ and $10 \mathrm{mM}$ Tris- $\mathrm{HCl} ; \mathrm{pH} 8.3)$, vortexed for ten seconds, and incubated at $55^{\circ} \mathrm{C}$ for an hour. The extracted DNA was incubated at $95^{\circ} \mathrm{C}$ for 10 minutes to inactivate the proteinase $\mathrm{K}$, and then stored at $-20^{\circ} \mathrm{C}$ until required.

\section{Detection of Plasmodium parasites by nPCR-HRM}

The extracted DNA was amplified using nested PCR, targeting $18 \mathrm{~S}$ rRNA genes in Plasmodium DNA. For the primary amplification step, forward (PL-1459-F: CTG
GTT AAT TCC GAT AAC), and reverse (PL-1706-R: TAA ACT TCC TTG TGT TAG AC) primers were designed using Primer3 software [34]. The second pair of primers used for the nested amplification reaction were PL-1473-Forward (3'-TAA CGA ACG AGA TCT TAA5') and PL-1679-Reverse (3'-GTT CCT CTA AGA AGC TTT-5') primers targeting a 204-270 base pair fragment (depending on the Plasmodium species) of a polymorphic region in the 18S rRNA gene [28]. Ten-fold dilutions of the primary PCR products were used as templates for the nested reactions. Each of the two amplification reactions were carried out in $10 \mu \mathrm{L}$ final reaction volumes consisting of $1 \mu \mathrm{L}$ DNA template, $2 \mu \mathrm{L}$ Hot Firepol ${ }^{\circ}$ HRM mix kit (Solis BioDyne, Estonia), $0.5 \mu \mathrm{L}$ of $0.5 \mu \mathrm{M}$ of both primers and $6 \mu \mathrm{L}$ nuclease free PCR water. The touchdown PCR thermal conditions consisted of an initial denaturation at $95^{\circ} \mathrm{C}$ for 5 minutes, 45 cycles of denaturation at $94^{\circ} \mathrm{C}$ for 20 seconds, decreasing annealing temperatures from $65^{\circ} \mathrm{C}$ to $50^{\circ} \mathrm{C}$ for 25 seconds (cycles $1-5$ ), $50^{\circ} \mathrm{C}$ for 40 seconds (cycles $6-10$ ), $50^{\circ} \mathrm{C}$ for 50 seconds (cycles $11-45$ ), and extension at $72^{\circ} \mathrm{C}$ for 30 seconds. A final extension of $72^{\circ} \mathrm{C}$ for 3 minutes was included before HRM analysis. Upon completion, the nested PCR process was transitioned into the melting phase (HRM) in the same closed tube system yielding distinct melting profiles in a Rotor-Gene $\mathrm{Q}^{\circ}$ machine (QIAGEN, Germany). The set of conditions for HRM included $0.2^{\circ} \mathrm{C}$ incremental temperature increases from $75^{\circ} \mathrm{C}$ to $90^{\circ} \mathrm{C}$, with fluorescence acquisition at the end of each 2 second temperature increment. DNA extracts from P. falciparum infected blood and deionized water were used as positive and negative controls, respectively. Products that produced curves distinct from the $P$. falciparum positive control were sequenced by Macrogen (Seoul, Korea) and aligned with reference sequences for Plasmodium malariae [GenBank: AB489193] and Plasmodium ovale [GenBank:AJ001527] using Geneious 6.1.6 software [35]. Protocols for nPCR [27] and APCR-HRM [28] were also separately used on all samples for comparison purposes.

\section{Validation of $\mathrm{nPCR}$-HRM sensitivity in comparison to established nPCR and APCR-HRM protocols}

To compare the detection limits of the three assays, we used the WHO International Standard for P. falciparum DNA, a standardized freeze-dried preparation of $P$. falciparum infected whole blood obtained from the National Institute for Biological Standards and Control (NIBSC; Hertfordshire, UK). Following NIBC recommendations, the lyophilized material was suspended in $500 \mu \mathrm{L}$ of sterile, nuclease-free water to a final concentration of $1 \times 10^{9} \mathrm{IU} /$ $\mathrm{mL}$, estimated to be 469,920 parasites/ $\mu \mathrm{L}$ [36]. To establish and compare each assay's LoD, we serially diluted the suspension in fresh uninfected whole blood. The sensitivity of 
each assay was tested using two replicate extractions of single-log (ten-fold) serial dilutions for the first four dilution points and four replicate extractions of each of the following eight half-log (3.16-fold) serial dilutions. The lowest concentration of DNA that tested positive in all replicates of a particular assay was set as the LoD. To narrow down the LoD further, additional tests were performed on half$\log$ and two-fold dilutions of the lowest concentration that could be consistently detected among the original serial dilution series. To assess the rate of possible false positives due to cross-contamination during the nested amplification step of nPCR-HRM, 20 negative controls, including eight extraction negative controls, were distributed in every third position, such that each DNA standard serial dilution amplification was adjacent to a negative control reaction.

\section{Statistical methods}

McNemar's Chi-Square tests were used to compare the sensitivity of nPCR-HRM to nPCR and APCR-HRM in detecting parasitaemia. Continuous/Gaussian data were compared using t-tests while Cohen's Kappa was calculated to compare the agreement between the results obtained using the different methods. All statistical inferences were drawn on two-tailed distributions with $\alpha=0.05$. The data were analysed using R 3.0.1 software [37].

\section{Results}

\section{Characteristics of study subjects}

While 5,672 and 1,412 patients tested positive for malaria at Sena and Tom Mboya Health Centres, respectively, during the study period, 197 subjects with undiagnosed febrile illness were enrolled into the study. Subjects were between 12 and 70 years of age with a mean and median age of 31.9 and 28.0 years, respectively. There were 114 females and 81 males, with the gender of two individuals not reported. About $53.3 \%(n=105)$ of the subjects were recruited from Sena Health Centre between Oct 15, 2012 and Oct 15, 2013 and the rest $(\mathrm{n}=92)$ from Tom Mboya Health Centre between May 28, 2012 and Feb 18, 2013. Although 7,553 and 2,378 patients tested negative for malaria at Sena and Tom Mboya Health Centres, respectively, only two smear/RDT-negative patients from each clinic were included in the study every week to minimize timebased selection bias. The mean age for the subjects recruited from Sena Health Centre was 29.1 years old. This population was significantly younger than that recruited from Tom Mboya Health Centre, which averaged 35.4 years old $(\mathrm{t}=3.3, \mathrm{df}=147.53, \mathrm{p}=0.001)$.

\section{Sensitivity comparison of the three techniques using serial} dilutions of the WHO Plasmodium DNA International Standard The LoD of the coupled nPCR-HRM technique was 236 parasites/mL, whereas the LODs of dPCR-HRM and
nPCR were 1,490 parasites $/ \mathrm{mL}$ and 4,700 parasites $/ \mathrm{mL}$, respectively.

Malaria and $P$. falciparum prevalence as generated by the three techniques

Prevalence estimates of Plasmodium parasitaemia generated by our nPCR-HRM technique relative to those generated through nPCR or APCR-HRM techniques are summarized in Figure 1. Results obtained by nPCR-HRM were in substantial agreement with those obtained by dPCR-HRM (Kappa $=0.699 ; 95 \%$ CI, 0.589-0.809) and fair agreement with those obtained by nPCR (Kappa $=0.395$; 95\% CI, 0.266-0.524). Nonetheless, our novel nPCR-HRM method detected significantly more cases of overall Plasmodium infections than did either dPCR-HRM $\left(\chi_{1}^{2}=\right.$ 21.04, $\mathrm{p}<0.001)$ or $\mathrm{nPCR}\left(x_{1}^{2}=40.02, \mathrm{p}<0.001\right)$. More specifically, nPCR-HRM also detected significantly more cases of $P$. falciparum infections than did either dPCR-HRM $\left(\chi_{1}^{2}=22.04, \mathrm{p}<0.001\right)$ or $\mathrm{nPCR}\left(\mathrm{\chi}_{1}^{2}=34.02, \mathrm{p}<0.001\right)$.

Based on nPCR-HRM analyses, 62 (31.5\%; 95\% CI, 25.038.0) of the 197 subjects enrolled in the study had Plasmodium parasites in their samples, of which 53 (26.9\%; 95\% CI, 20.7-33.1) had P. falciparum parasites. While P. falciparum prevalence did not significantly differ between the two sampling sites during the study period $(\mathrm{p}=0.137)$, the overall Plasmodium spp. prevalence was greater in samples collected at Tom Mboya Health Centre than those collected at Sena Health Centre $(\mathrm{p}=0.013)$. Samples with Plasmodium collected at Tom Mboya Health Centre also had higher Plasmodium species diversity than those collected at Sena Health Centre. Samples collected at Tom Mboya Health Centre included 26 (72.2\%) P. falciparum only infections, five (13.9\%) P. malariae only infections, two $(5.6 \%)$ P. ovale only infections, two $(5.6 \%)$ double infections of $P$. falciparum and $P$. ovale, and one (2.8\%)

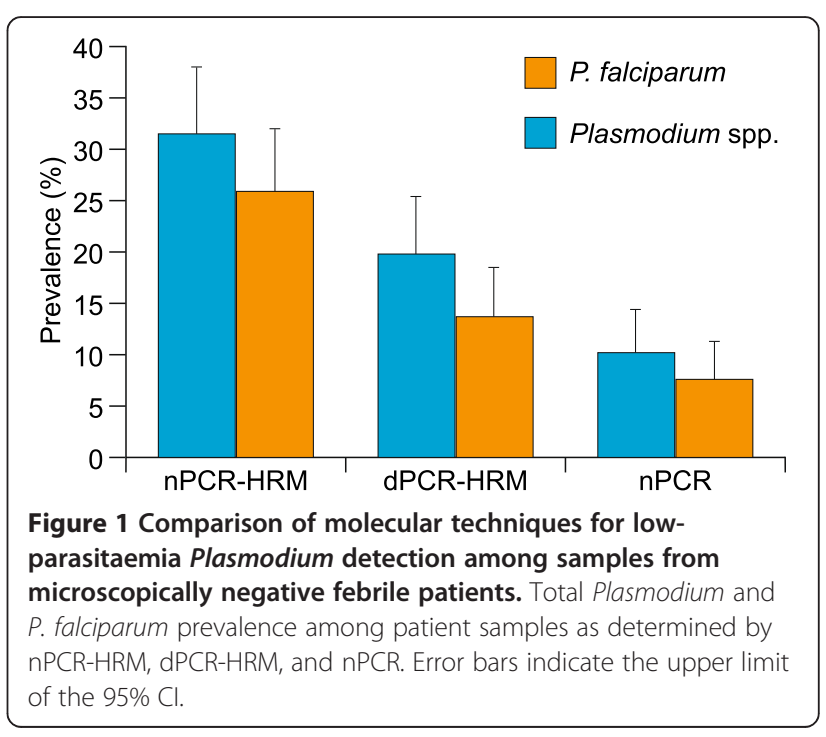




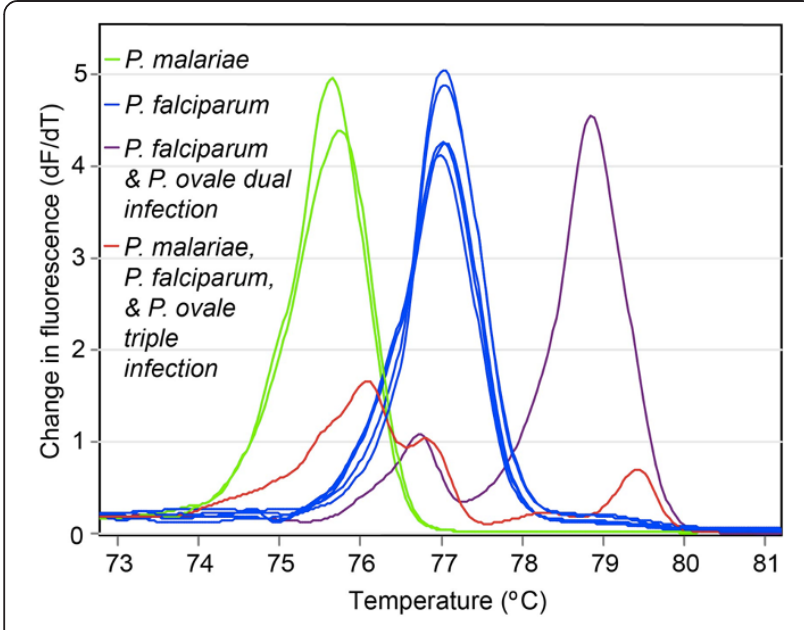

Figure 2 Representative Plasmodium HRM profiles from a subset of the study samples. Distinct melting profiles, represented by change in fluorescence units with increasing temperatures $(\mathrm{df} / \mathrm{dt}$ ) of patient samples with $P$. malariae, $P$. falciparum, and $P$. ovale infections.

triple infection of $P$. falciparum, $P$. malariae, and $P$. ovale. Samples collected at Sena Health Centre had only two $P$. ovale infections with the remaining 24 infections being $P$. falciparum. The unique HRM profiles of the three Plasmodium parasites are represented in Figure 2. Based on dPCR-HRM, only 39 (19.8\%; 95\% CI, 14.2-25.4) of the patient samples had detectable Plasmodium parasites, among which 27 (13.7\%; 95\% CI, 8.9-18.5) were P. falciparum infections. nPCR revealed malaria parasites in only 20 (10.2\%; 95\% CI, 5.9-14.4) of the samples, among which 15 (7.6\%; 95\% CI, 3.9-11.3) were P. falciparum infections. No mixed infections were detected by either nPCR or dPCR-HRM. To verify that the nested amplification approaches did not yield false positives due to crosscontamination, these assays were repeated with the same samples in three successive runs, all of which produced identical results for the individual samples.

\section{Anti-malarial drug prescription patterns}

Among the 197 patients presenting with febrile illness enrolled in this study, $152(77.2 \%)$ were treated with anti-malarial drugs. While $42(67.7 \%)$ of the 62 patients with detectable Plasmodium parasites were treated with anti-malarial drugs, 110 (81.5\%) of the 135 patients without detectable Plasmodium parasites were incorrectly treated with anti-malarial drugs. However, these patterns differed between the two Health Centres, as represented in Figure 3. While in Tom Mboya Health Centre, only $16(44.4 \%)$ of the 36 patients with detectable Plasmodium parasites were treated with anti-malarial drugs, all 26 malaria patients with detectable parasites at Sena Health Centre were treated with anti-malarials. Yet, among the patients that did not have Plasmodium

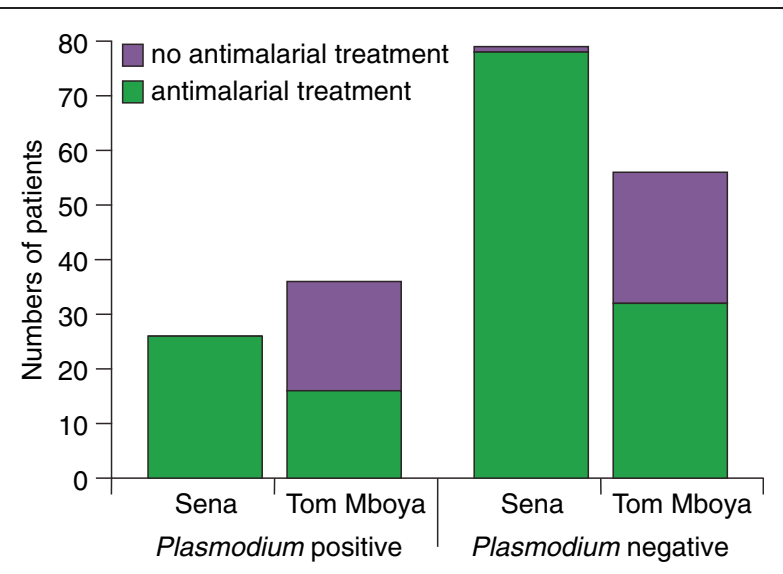

Figure 3 Anti-malarial treatment of febrile patients with and without low parasitaemia Plasmodium infections identified by nPCR-HRM. Numbers of patients treated or not treated with anti-malarial drugs in Sena and Tom Mboya Health Centres.

infection, more than half were treated with anti-malarial drugs at both Tom Mboya (32 out of 56, 57.1\%) and Sena Health Centres (78 out of 79, 98.7\%).

\section{Discussion}

By combining the high sensitivity of nested PCR with real-time species differentiating HRM analysis, the reporting of Plasmodium infection prevalence that includes low-parasitaemia infections can be synergistically enhanced to improve size estimates of its infectious human reservoir in regions where malaria elimination efforts are being scaled up. Indeed, nPCR-HRM was able to detect $P$. falciparum at orders of magnitude lower concentrations than either nPCR or dPCR-HRM. The LoD of the coupled nPCR-HRM assay (236 parasites/ $\mathrm{mL}$ ) is however in the same range as TaqMan Probe assays validated using the same $P$. falciparum DNA standard (313 parasites $/ \mathrm{mL}$ ) [36]. Out of the 62 patient samples in which Plasmodium parasites were detected by the novel nPCR-HRM technique, the well-established nPCR [27] and dPCR-HRM [28] molecular techniques detected Plasmodium parasites in only 20 and 39 samples, respectively. The same is evident for $P$. falciparum, where nPCR-HRM identified $26.9 \%$ prevalence among microscopically negative febrile illness patients, while nPCR and APCR-HRM identified only $10.2 \%$ and $13.7 \%$ prevalence, respectively. These results are consistent with the lower sensitivity of nPCR previously reported in comparison to APCR-HRM [38], yet further demonstrate that nPCR-HRM is significantly more sensitive and specific than either nPCR or APCR-HRM, and was also the only method able to detect double and triple infections with distinct Plasmodium parasites.

Although other novel molecular methods for Plasmodium detection have been reported recently, they either 
failed to undercut the detection thresholds of microscopy on clinical specimen [39] or were limited in ability to differentiate Plasmodium species $[25,36]$. Due to these limitations in detecting and differentiating low-parasitaemia Plasmodium infections, no previous studies have assessed drug administration among subjects with malaria parasitaemia below the detection limits of standard microscopy and RDT.

The lack of diagnostic tools sufficiently sensitive to detect low Plasmodium parasitaemia in rural clinics in malaria endemic regions of SSA, and that can differentially diagnose other non-malaria febrile illnesses, including bacterial, mycobacterial, fungal, and arboviral infections, is a big challenge [12,20-24]. While limited training of laboratory technicians has been suggested as a cause of poor malaria diagnoses [40], clinicians are left with little option, as a result of diagnostic limitations in rural clinics, but to treat patients with anti-malarials and antibiotics based on clinical symptoms [6,7,20-22].

Febrile illness misdiagnosis can be significant in malaria endemic settings, resulting in a myriad of downstream issues including improper treatment, chronic suffering of patients as the underlying cause of illness remains unknown, and drug wastage [7]. Moreover, poor malaria diagnostics can undermine the diagnosis of non-malarial febrile illnesses $[10,23,24]$. Due to the limitations of microscopy and RDTs in detecting low-parasitaemia malaria $[14,15]$ and clinical heuristic treatment practices [6,7,20-22], 197 febrile patients from both study sites were diagnosed as not having malaria, but were still given different medications on the basis of clinical signs. In endemic regions with high transmission intensity, such presumptive fever management may be justifiable, as test-based management may not be safe or cost effective [10]. Because clinicians are limited to heuristic methods of clinical diagnoses in such cases, treatment patterns are likely to differ between clinics and clinicians. Indeed, while all low Plasmodium parasitaemia cases at Sena Health Centre were treated with antimalarial drugs, only $44.4 \%$ of low-parasitaemia patients at Tom Mboya Health Centre were treated with anti-malarial drugs. However, in both Health Centres, the majority of anti-malarial drugs were prescribed to patients in whom we did not detect Plasmodium parasitaemia.

The discrepant prescribing patterns between the two study sites may be due to the over three times greater numbers of patients with microscopically positive malaria observed at Sena Health Centre than at Tom Mboya Health Centre during the respective sampling periods. Accessibility to other health facilities may also have influenced prescribing practices. Compared to Tom Mboya Health Centre on Rusinga Island, Sena Health Centre on Mfangano Island is more isolated from the mainland where patients access other nearby and specialized health centres that they may be referred to for assessment of differential diagnoses. Due to the relatively limited accessibility to such referral clinics for patients on Mfangano Island, clinicians may have had a stronger tendency to prescribe the more readily available anti-malarials at Sena Health Centre.

Individuals with extremely low-parasitaemia Plasmodium infection are likely to have endemic stability to malaria infection and may be able to tolerate a low degree of parasitaemia with absence of clinical disease [19]. Therefore, anti-malarial drugs do not treat febrile illness patients with sub-microscopic Plasmodium infections for the underlying differential diagnosis of their febrile symptoms [10]. Nonetheless, treatment of patients with sub-microscopic Plasmodium infection, may contribute to integrated malaria elimination/control efforts by reducing the size of the infectious reservoir within communities [9-11].

This study demonstrates how improper febrile illness diagnoses confound prevalence estimates of Plasmodium infections and other differential diagnoses. Some nonmalaria febrile patients are diagnosed and treated for malaria based on their clinical symptoms, and may be inadvertently integrated into epidemiological statistics [3-6], while some low-parasitaemia Plasmodium infections go unrecognized [2]. As a result, malaria burden and infection estimates suffer from unknown large margins of error [4]. The complementary use of sensitive and specific molecular based methods informs size estimates of the potential infectious reservoir of malaria in endemic regions, while also providing estimates of undifferentiated febrile illness burden.

Improved understanding of Plasmodium infection rates will translate into the development of better rural malaria diagnostics and treatments, ultimately reducing gametocyte incidence rates in asymptomatic populations with low-parasitaemia malaria [12]. While individuals with submicroscopic parasitaemia have significantly lower Plasmodium transmission rates to mosquito vectors [41,42], they may still constitute a considerable proportion of the human infectious reservoir $[12,42,43]$, a phenomenon that probably prevails among individuals with endemic stability to malaria infection in the endemic study sites. Therefore, more accurate parasite-detection based diagnosis and treatment may reduce malaria transmission [42]. Lowparasitaemia malaria can result in acute febrile illness [5] and individuals that may have low-parasitaemia in the peripheral blood may still require anti-malarial treatment due to higher parasite loads that can still be sequestered in capillaries [44]. In contrast, acute febrile illnesses have many aetiologies ranging from bacterial, fungal and mycobacterial infections to arboviral infections [24]. These unknowns complicate appropriate febrile illness diagnosis and treatment. Clear guidelines and policies on the management of non-malaria acute febrile illnesses are required urgently, particularly in malaria endemic regions. 
This study's approach to low-parasitaemia Plasmodium detection and species differentiation is a valuable molecular tool that can be used to complement microscopy and RDT and enhance understanding of specific Plasmodium infection rates. While sensitive and specific, nPCRHRM was not designed as a diagnostic tool for health clinics, as it requires real-time PCR infrastructure, specialized training to avoid the cross-contamination risks associated with nested PCR assays, and more man power in comparison to RDTs. Recent developments in both loop-mediated isothermal amplification (LAMP) [25] and isothermal lateral flow-recombinant polymerase amplification (LF-RPA) [26] assays show promise as true point-of-care tests with high specificity and sensitivity for detecting low-parasitaemia Plasmodium infections. Nonetheless, nPCR-HRM can be employed for monitoring low-parasitaemia infection rates within malaria endemic regions to assess the progress of regional malaria elimination efforts.

\section{Conclusions}

This is the first study that employs low-parasitaemia Plasmodium diagnostics to quantify both the overprescription of anti-malarial drugs in non-malaria febrile patients and under-prescription of anti-malarial drugs in low-parasitaemia malaria patients. While patient febrile illness symptoms cannot be attributed to any of the submicroscopic Plasmodium infections identified in this study, consistent anti-malarial treatment limited to such cases should contribute to regional malaria elimination efforts [9-11]. Other recent cohort studies have contrasted presumptive clinical diagnoses and anti-malarial drug treatments with malaria parasitaemia detectable by microscopy [21,23,24], as microscopy [8], alongside RDTs [5], is the mainstay of present malaria diagnostic practices in most malaria endemic regions of Africa. In a low transmission area clinical cohort study in Tanzania, Reyburn and colleagues found that $38 \%$ of suspected malaria patients were treated for malaria without further testing and $48 \%$ of microscopically malaria negative febrile patients were presumptively treated with anti-malarial drugs [21]. More recently, Crump and colleagues showed that only $2.7 \%$ of patients diagnosed with malaria in two hospitals in northern Tanzania were actually malaria positive as determined by microscopy [24]. Considering Plasmodium infection prevalence, we further demonstrate the inadequacy of rural diagnostics in detecting low-parasitaemia infections.

While patients with undiagnosed and untreated lowparasitaemia Plasmodium infections may not suffer from acute febrile illness as a result of malaria, they may contribute to a significant proportion of the infectious reservoir during their asymptomatic state. Conversely, the lack of diagnostics and treatment for unrecognized differential febrile illness diagnoses may have severe health implications that cannot be addressed properly. Epidemiological estimates need to take into account the considerable proportions of asymptomatic low-parasitaemia Plasmodium infections that contribute to the infectious reservoir, while also considering the health impacts of unrecognized differential diagnoses of febrile illness in malaria endemic regions.

\section{Abbreviations}

PCR: Polymerase chain reaction; HRM: High resolution melting; nPCR: Nested Polymerase chain reaction; nPCR-HRM: nested Polymerase chain reaction - high resolution melting; $\mathrm{dPCR}-\mathrm{HRM}$ : Direct Polymerase chain reaction - high resolution melting; RDT: Rapid diagnostic test; WHO: World Health Organization; SSA: sub-Saharan Africa; KEMRI: Kenya Medical Research Institute; DDSR: Division of Disease Surveillance and Response; Cl: Confidence Interval; LAMP: Loop-mediated isothermal amplification; LF-RPA: Lateral flow-recombinant polymerase amplification; LoD: Limits of detection.

\section{Competing interests}

The authors declare that they have no competing interests.

\section{Authors' contributions}

PNK carried out the molecular assay optimization, data collection and analysis, and drafted the manuscript. DOO facilitated sample and data collection. POM helped draft the manuscript. PS facilitated sample collections. DKM contributed to the initial idea of the study and helped draft the paper. JV contributed to the initial idea of the study, designed the methodology, analysed data, validated the assays and wrote the manuscript. All authors read and approved the final manuscript.

\section{Acknowledgements}

We thank all study participants, clinicians and lab technicians of Tom Mboya (Lencer Ongola, and Hillary Tabut) and Sena (Paul Ombonya and Harry Songa) Health Centres, Martin Koome Mbaya for his help and facilitation in the laboratory, Benedict Orindi and Thomas Gachie for assistance with Statistical analyses and the entire ML-EID laboratory fraternity for facilitating the project in one way or the other. Additionally, we would like to thank Prof. Clifford Muteru and Dr. Baldwyn Torto for providing helpful comments on the manuscript. This study was mainly funded by the Swedish International Development Cooperation Agency (SIDA), supplemented by funds from Training Health Researchers into Vocational Excellence (THRiVE) in East Africa (grant number 087540) funded by Wellcome Trust. The article's contents are solely the responsibility of the authors and do not necessarily represent the official views of the supporting offices.

\section{Author details}

'Martin Lüscher Emerging Infectious Diseases (ML-EID) Laboratory, Molecular Biology and Bioinformatics Unit, International Centre of Insect Physiology and Ecology (icipe), P.O. Box 30772, Nairobi 00100, Kenya. ${ }^{2}$ St. Jude's Clinic, International Centre of Insect Physiology and Ecology (icipe), P.O. Box 30, Mbita 40305, Kenya. ${ }^{3}$ Department of Biochemistry and Molecular Biology, Egerton University Njoro Campus, P.O. Box 536, Egerton 20115, Kenya. ${ }^{4}$ Department of Medical Biosciences, University of Western Cape, Private Bag X17, Bellville 7535, Republic of South Africa. ${ }^{5}$ Yale School of Public Health, Yale University, 60 College Street, New Haven, CT 06510, USA.

Received: 11 June 2014 Accepted: 5 November 2014

Published: 15 November 2014

\section{References}

1. World Health Organization: World Malaria Report 2013. Geneva: World Health Organization; 2013.

2. Murray CJL, Rosenfeld LC, Lim SS, Andrews KG, Foreman KJ, Haring D, Fullman N, Naghavi M, Lozano R, Lopez AD: Global malaria mortality between 1980 and 2010: a systematic analysis. Lancet 2012, 379:413-431.

3. Hommel M: Towards a research agenda for global malaria elimination. Malar J 2008, 7:S1 
4. Lynch M, Korenromp E, Eisele T, Newby H, Steketee R, Kachur SP, Nahlen B, Bhattarai A, Yoon S, MacArthur J, Newman R, Cibulskis R: New global estimates of malaria deaths. Lancet 2012, 380:559.

5. Chinkhumba J, Skarbinski J, Chilima B, Campbell C, Ewing V, San Joaquin M, Sande J, Ali D, Mathanga D: Comparative field performance and adherence to test results of four malaria rapid diagnostic tests among febrile patients more than five years of age in Blantyre, Malawi. Malar J 2010, 9:209.

6. Roucher C, Rogier C, Dieye-Ba F, Sokhna C, Tall A, Trape J-F: Changing malaria epidemiology and diagnostic criteria for Plasmodium falciparum clinical malaria. PLoS One 2012, 7:e46188.

7. Amexo M, Tolhurst R, Barnish G, Bates I: Malaria misdiagnosis: effects on the poor and vulnerable. Lancet 2004, 364:1896-1898.

8. Icke G, Davis R, McConnell W: Teaching health workers malaria diagnosis. PLOS Med 2005, 2:e11.

9. Harris I, Sharrock WW, Bain LM, Gray K-A, Bobogare A, Boaz L, Lilley K, Krause D, Valley A, Johnson M-L, Gatton ML, Shanks GD, Cheng Q: A large proportion of asymptomatic Plasmodium infections with low and sub-microscopic parasite densities in the low transmission setting of Temotu Province, Solomon Islands: challenges for malaria diagnostics in an elimination setting. Malar J 2010, 9:254.

10. Bisoffi Z, Gobbi F, Buonfrate D, Van den Ende J: Diagnosis of Malaria Infection with or without Disease. Mediterr J Hematol Infect Dis 2012, 4:e2012036.

11. McMorrow ML, Aidoo M, Kachur SP: Malaria rapid diagnostic tests in elimination settings-can they find the last parasite? Clin Microbiol Infect 2011, 17:1624-1631.

12. Okell LC, Ghani AC, Lyons E, Drakeley CJ: Submicroscopic infection in Plasmodium falciparum-endemic populations: a systematic review and meta-analysis. J Infect Dis 2009, 200:1509-1517.

13. Shakely D, Elfving $K$, Aydin-Schmidt B, Msellem Ml, Morris U, Omar R, Weiping X Petzold M, Greenhouse B, Baltzell KA, Ali SA, Björkman A, Mårtensson A: The usefulness of rapid diagnostic tests in the new context of low malaria transmission in Zanzibar. PLoS One 2013, 8:e72912.

14. Payne D: Use and limitations of light microscopy for diagnosing malaria at the primary health care level. Bull World Health Organ 1988, 66:621-626.

15. Wongsrichanalai C, Barcus MJ, Muth S, Sutamihardja A, Wernsdorfer WH: A review of malaria diagnostic tools: microscopy and rapid diagnostic test (RDT). Am J Trop Med Hyg 2007, 77:119-127.

16. Joanny F, Löhr SJZ, Engleitner T, Lell B, Mordmüller B: Limit of blank and limit of detection of Plasmodium falciparum thick blood smear microscopy in a routine setting in Central Africa. Malar J 2014, 13:234.

17. Khairnar K, Martin D, Lau R, Ralevski F, Pillai DR: Multiplex real-time quantitative $P C R$, microscopy and rapid diagnostic immuno-chromatographic tests for the detection of Plasmodium spp: performance, limit of detection analysis and quality assurance. Malar J 2009, 8:284.

18. Bisoffi Z, Sirima SB, Menten J, Pattaro C, Angheben A, Gobbi F, Tinto H, Lodesan C, Neya B, Gobbo M, Van den Ende J: Accuracy of a rapid diagnostic test on the diagnosis of malaria infection and of malaria-attributable fever during low and high transmission season in Burkina Faso. Malar J 2010, 9:192.

19. McGuinness D, Koram K, Bennett S, Wagner G, Nkrumah F, Riley E: Clinical case definitions for malaria: clinical malaria associated with very low parasite densities in African infants. Trans R Soc Trop Med Hyg 1998, 92:527-531.

20. Joshi R, Colford JM, Reingold AL, Kalantri S: Nonmalarial acute undifferentiated fever in a rural hospital in central India: diagnostic uncertainty and overtreatment with antimalarial agents. Am J Trop Med Hyg 2008, 78:393-399.

21. Reyburn H, Ruanda J, Mwerinde O, Drakeley C: The contribution of microscopy to targeting antimalarial treatment in a low transmission area of Tanzania. Malar J 2006, 5:4.

22. Chandler CIR, Jones C, Boniface G, Juma K, Reyburn H, Whitty CJM: Guidelines and mindlines: why do clinical staff over-diagnose malaria in Tanzania? A qualitative study. Malar J 2008, 7:53.

23. Hertz JT, Munishi OM, Ooi EE, Howe S, Lim YL, Chow A, Morrissey AB, Bartlett JA Onyango JJ, Maro VP, Kinabo GD, Saganda W, Gubler DJ, Crump JA: Chikungunya and dengue fever among hospitalized febrile patients in northern Tanzania. Am J Trop Med Hyg 2012, 86:171-177.

24. Crump JA, Morrissey AB, Nicholson WL, Massung RF, Stoddard RA, Galloway RL, Ooi EE, Maro VP, Saganda W, Kinabo GD, Muiruri C, Bartlett JA: Etiology of severe non-malaria febrile illness in Northern Tanzania: a prospective cohort study. PLoS Negl Trop Dis 2013, 7:e2324.

25. Hopkins HP, González IJ, Polley SD, Angutoko P, Ategeka J, Asiimwe C, Agaba B, Kyabayinze DJ, Sutherland CJ, Perkins MD, Bell D: Highly sensitive detection of malaria parasitemia in an endemic setting: Performance of a new LAMP kit in a remote clinic in Uganda. J Infect Dis 2013 208:645-652.

26. Kersting S, Rausch V, Bier FF, von Nickisch-Rosenegk M: Rapid detection of Plasmodium falciparum with isothermal recombinase polymerase amplification and lateral flow analysis. Malar J 2014, 13:99.

27. Snounou G, Viriyakosol S, Zhu XP, Jarra W, Pinheiro L, do Rosario VE, Thaithong S, Brown KN: High sensitivity of detection of human malaria parasites by the use of nested polymerase chain reaction. Mol Biochem Parasitol 1993, 61:315-320.

28. Mangold KA, Manson RU, Koay ESC, Stephens L, Regner MA, Thomson RB Jr, Peterson LR, Kaul KL: Real-time PCR for detection and identification of Plasmodium spp. J Clin Microbiol 2005, 43:2435-2440.

29. GoK: Suba District PRSP: Consultation Report for the period 2001-2004. Nairobi, Kenya: Government of the Republic of Kenya, Ministry of Finance and Planning; 2001:309.

30. Minakawa N, Mutero CM, Githure JI, Beier JC, Yan G: Spatial distribution and habitat characterization of anopheline mosquito larvae in Western Kenya. Am J Trop Med Hyg 1999, 61:1010-1016.

31. Kenya Ministry of Public Health and Sanitation: National guidelines for the diagnosis, treatment and prevention of malaria in Kenya. Nairobi: Division of Malaria Control, Ministry of Public Health and Sanitation; 2010.

32. Gouagna LC, Okech BA, Kabiru EW, Killeen GF, Obare P, Ombonya S, Bier JC, Knols BG, Githure JI, Yan G: Infectivity of Plasmodium falciparum gametocytes in patients attending rural health centres in western Kenya. East Afr Med J 2003, 80:627-634.

33. Kawasaki ES: Sample preparation from blood, cells, and other fluids. In PCR protocols: A guide to methods and applications. Edited by Innis MA, Gelfand DH, Sninsky JJ, White TJ. New York: Academic Press; 1990:146-152.

34. Untergasser A, Cutcutache I, Koressaar T, Ye J, Faircloth BC, Remm M, Rozen SG: Primer3-new capabilities and interfaces. Nucleic Acids Res 2012, 40:e115.

35. Kearse M, Moir R, Wilson A, Stones-Havas S, Cheung M, Sturrock S, Buxton S, Cooper A, Markowitz S, Duran C, Thierer T, Ashton B, Meintjes P, Drummond A: Geneious Basic: an integrated and extendable desktop software platform for the organization and analysis of sequence data. Bioinformatics 2012, 28:1647-1649.

36. Alemayehu S, Feghali KC, Cowden J, Komisar J, Ockenhouse CF, Kamau E: Comparative evaluation of published real-time PCR assays for the detection of malaria following MIQE guidelines. Malar J 2013, 12:277.

37. R Development Core Team: $R$ : A language and environment for statistical computing. Vienna: R Foundation for Statistical Computing; 2010. http://www.R-project.org

38. Boonma P, Christensen PR, Suwanarusk R, Price RN, Russell B, Lek-Uthai U: Comparison of three molecular methods for the detection and speciation of Plasmodium vivax and Plasmodium falciparum. Malar J 2007, 6:124.

39. Reller ME, Chen WH, Dalton J, Lichay MA, Dumler JS: Multiplex 5' nuclease quantitative real-time PCR for clinical diagnosis of malaria and species-level identification and epidemiologic evaluation of malaria-causing parasites, including Plasmodium knowlesi. J Clin Microbiol 2013, 51:2931-2938.

40. Ngasala B, Mubi M, Warsame M, Petzold MG, Massele AY, Gustafsson LL, Tomson G, Premji Z, Bjorkman A: Impact of training in clinical and microscopy diagnosis of childhood malaria on antimalarial drug prescription and health outcome at primary health care level in Tanzania: a randomized controlled trial. Malar J 2008, 7:199.

41. Lin JT, Saunders DL, Meshnick SR: The role of submicroscopic parasitemia in malaria transmission: what is the evidence? Trends Parasitol 2014, 30:183-190.

42. Okell LC, Bousema T, Griffin JT, Ouédraogo AL, Ghani AC, Drakeley CJ: Factors determining the occurrence of submicroscopic malaria infections and their relevance for control. Nat Commun 2012, 3:1237.

43. Schneider P, Bousema JT, Gouagna LC, Otieno S, van de Vegte-Bolmer M, Omar SA, Sauerwein RW: Submicroscopic Plasmodium falciparum gametocyte densities frequently result in mosquito infection. Am J Trop Med Hyg 2007, 76:470-474.

44. Trampuz A, Jereb M, Muzlovic I, Prabhu RM: Clinical review: Severe malaria. Crit Care 2003, 7:315-323

\section{doi:10.1186/1475-2875-13-429}

Cite this article as: Kipanga et al:: High-resolution melting analysis reveals low Plasmodium parasitaemia infections among microscopically negative febrile patients in western Kenya. Malaria Journal 2014 13:429. 